\title{
A INSTITUIÇÃO ASILAR COMO FATOR POTENCIALIZADOR DA DISFAGIA
}

\section{The asylum as worsening factor for dysphagia}

\author{
Ana Maria Furkim ${ }^{(1)}$, Silvana Triló Duarte ${ }^{(2)}$, Patrícia Tenório Hildebrandt ${ }^{(3)}$, Katia Alonso Rodrigues ${ }^{(4)}$
}

\begin{abstract}
RESUMO
Objetivo: verificar se as condições referentes à estrutura geral dos asilos, recursos humanos e rotinas diárias de alimentação em instituições asilares, podem potencializar alterações da dinâmica da deglutição em idosos. Métodos: foi aplicado questionário aos dirigentes de cinco instituições asilares do município do Rio de Janeiro, no qual constavam perguntas em relação aos recursos materiais, humanos e rotina alimentar. Resultados: o asilo do sistema privado foi o único a aproximar-se das condições ideais da estrutura geral para o atendimento do idoso. Em relação aos recursos humanos


relação às rotinas diárias de alimentação, em uma das instituições pesquisadas, cuja maioria dos residentes não faziam uso de próteses dentárias, não havia restrição na consistência alimentar oferecida. Outro fator significativo diz respeito à negligência referente à higiene bucal, favorecendo a colonização de bactérias na cavidade oral, podendo agravar infecções pulmonares no caso de microaspirações. O fato dos idosos deitarem para dormir logo após o término das refeições, como ocorrido em três das instituições pesquisadas, tem grande importância na medida em que favorece a ocorrência de episódios de refluxo gastroesofágico. Conclusão: foram observados que em todas as instituições pesquisadas há fatores que podem potencializar um distúrbio de deglutição, como problemas em relação à estrutura geral e ou aos recursos humanos e ou relativos à rotina alimentar estabelecida.
\end{abstract}

DESCRITORES: Asilo; Idoso; Transtornos da Deglutição

\section{INTRODUÇÃO}

O envelhecimento pode ser considerado como mais uma etapa do desenvolvimento normal do

(1) Fonoaudióloga; Docente e Coordenadora do Curso de Especialização de Disfagia e Atuação no Âmbito Hospitalar do CEFAC - Pós-Graduação em Saúde e Educação, São Paulo, SP, Brasil; Docente do Programa de Pós-graduação em Distúrbios da Comunicação da Universidade Tuiuti do Paraná, UTP, Tuiuti, PR; Doutora em Distúrbios da Comunicação pela Universidade Federal de São Paulo. Diretora do IGD.

(2) Fonoaudióloga, Mestre em Disturbios da Comunicação Universidade Tuiuti do Paraná, Fonoaudióloga do Hospital Universitário do Oeste do Paraná, Docente Faculdade Assis Gurgacs_FAG

(3) Fonoaudióloga da Cuidar Home Care, Rio de Janeiro, RJ; Especialista em Motricidade Orofacial pelo CEFAC - PósGraduação em Saúde e Educação.

(4) Fonoaudióloga do Hospital São Paulo da Universidade Federal de São Paulo, UNIFESP, São Paulo, SP; Doutora em Distúrbios da Comunicação pela Universidade Federal de São Paulo.

Conflito de interesses: inexistente indivíduo. Todo este processo dinâmico e gradativo é marcado por alterações funcionais, bioquímicas, morfológicas e psicossociais, deixando o organismo mais suscetível às agressões extrínsecas e intrínsecas, o que interfere diretamente na função de adaptação do ser humano ao seu ambiente, repercutindo no estilo e na qualidade de vida dessa população ${ }^{1}$.

Não há idade limite que determine o início da ocorrência das primeiras mudanças. Além disso, essas mudanças acometem cada indivíduo de forma diferenciada, com características e intensidade particulares, cada qual com suas potencialidades e limitações ${ }^{2}$.

À medida que envelhecemos, a habilidade de deglutir de forma segura passa por mudanças anatômicas e fisiológicas que podem favorecer o risco de ocorrer uma deglutição incoordenada ${ }^{3}$.

Considerando as funções estomatognáticas, as alterações de deglutição são bastante freqüentes e significativas ${ }^{4-7}$. Estas podem gerar complicações como desidratação e má nutrição, 
ocasionando isolamento e depressão nos idosos. Além disso, a incoordenação da deglutição (com favorecimento da aspiração traqueal) é um dos principais fatores responsáveis por pneumonias em idosos ${ }^{8}$. Conseqüentemente estas alterações podem contribuir para o aumento dos índices de morbidade 4, 9-11. Uma das justificativas para essas alterações é a ocorrência do declínio no controle do sistema neuromuscular, podendo resultar em prejuízo de qualquer uma das fases da deglutição ${ }^{12}$

Frente a todas essas mudanças naturais referentes à deglutição, é fundamental que as instituições asilares estejam preparadas para atender essa população. É necessário conhecimento específico dos profissionais envolvidos, sendo importante a capacitação profissional e a educação continuada de toda a equipe interdisciplinar que atua diretamente com os idosos institucionalizados ${ }^{12,13}$.

A preocupação com a nutrição e hidratação adequadas nessa população deve ser uma constante, pois a diminuição de ingestão alimentar nos asilos é observada com freqüência ${ }^{3} e$ tem como um dos principais fatores etiológicos os distúrbios da deglutição.

Atualmente, muitos idosos desprovidos de seus familiares procuram as instituições asilares pela certeza de estarem em um local onde serão cuidados por profissionais capacitados, garantindo sua segurança e saúde. Contudo, uma das realidades observadas em muitos lugares é a falta de qualificação dos recursos humanos ${ }^{14}$.

Dentro do ambiente asilar pode haver grande desconhecimento sobre fatores de risco para disfagia e aspiração, o que aumenta o risco a saúde dos residentes. Isso é particularmente importante quando se sabe que grande parte dos episódios de aspiração nos idosos é silenciosa, ou seja, sem sinais clínicos imediatos evidentes (tosse) ${ }^{15}$, tornando mais difícil a percepção do problema.

Outra questão importante diz respeito à perda da identidade e liberdade imposta pelo regime asilar sendo algo bastante significativo, na medida em que precisam se adaptar à rotina do local. Os hábitos de higiene oral, fundamentais a manutenção da cavidade oral sadia, por vezes são negligenciados ${ }^{14,16,17}$.

Diante dessa problemática, o objetivo do presente estudo foi verificar se as condições referentes à estrutura geral, recursos humanos e rotinas diárias de alimentação das instituições asilares pesquisadas podem ser potencializadores de alterações na dinâmica da deglutição dos idosos.

A partir desses achados, busca-se reforçar a importância de promover políticas de orientação dos asilos sobre as adaptações necessárias para promoção da qualidade de vida dos idosos, garantindo principalmente a alimentação de forma segura.

\section{MÉTODOS}

O presente estudou caracterizou-se pela análise qualitativa por meio do levantamento dos dados em campo. A coleta da amostra foi realizada durante $o$ mês de novembro de 2006. O pesquisador visitou cinco instituições asilares no município do Rio de Janeiro, sendo quatro filantrópicas e uma de caráter privado.

A escolha das instituições foi aleatória, baseada em uma lista de asilos obtida por meio de um catálogo de instituições registrado na universidade aberta da terceira idade da Universidade do Estado do Rio de Janeiro. Após a escolha, o pesquisador entrou em contato por telefone com os dirigentes dos asilos para verificar a possibilidade da realização da pesquisa.

Os responsáveis por cada instituição responderam oralmente, no mesmo dia da visita, um questionário adaptado de Wakasugi e colaboradores ${ }^{15}$ (Figura 1). O questionário abrangeu os seguintes tópicos:

A) Identificação: caráter da instituição; critério de elegibilidade para aceitação dos idosos (sexo, idade e nível de independência);

B) Recursos humanos disponíveis (médico, enfermeiro, assistente social, psicólogo, terapeuta ocupacional, nutricionista, fisioterapeuta, fonoaudiólogo e outros);

C) Rotinas diárias como higiene oral e sono; alimentação propriamente dita, especificando a consistência da dieta, quantidade oferecida, preocupação com excesso de sal ou açúcar, local das refeições, postura durante e após alimentação e prazer alimentar.

A pesquisa foi avaliada pelo Comitê de Ética e Pesquisa (CEP) do CEFAC - Pós-Graduação em Saúde e Educação, aprovada sob o número 133/06. Os dirigentes das instituições leram e assinaram o Termo de Consentimento Livre e Esclarecido.

\section{RESULTADOS}

Com o objetivo de descrever os resultados, as cinco instituições pesquisadas foram identificadas pelas letras A, B, C, D e E, não sendo empregados testes estatísticos quanto ao resultado. (Figura 2) 


\section{Protocolo de avaliação para instituições asilares}

Nome da instituição:

Endereço:

Telefone:

Responsável:

1. Caráter da instituição:

Privada ( ) Filantrópica ( ) Convênio com a prefeitura ( )

2. Número de idosos:

\section{Critério de elegibilidade:}

3.1. sexo:

$\mathrm{F}(\mathrm{)} \quad \mathrm{M}(\mathrm{)} \quad \mathrm{F} / \mathrm{M}(\mathrm{)}$

3.2. Idade mínima:

\subsection{Nível de dependência:}

dependentes ( ) semi-dependentes ( ) independentes ( )

\section{Regime:} fechado ( ) aberto ( ) depende do idoso ( )

5. Recursos humanos disponíveis:
Médico ( ) - Freqüência:
- Especialidade médica:
Enfermeiro ( ) - Freqüência:
- Especialidade:
Assistente social ( ) - Freqüência:
- Especialidade:
Psicólogo ( ) - Freqüência
- Especialidade:
Nutricionista ( ) - Freqüência:
Fonoaudiólogo ( ) - Freqüência:
- Especialidade:
Fisioterapeuta ( ) - Freqüência:
- Especialidade:
Terapeuta ocupacional ( ) - Freqüência:
- Especialidade:
- Especialidade:

Voluntários ( ) - Freqüência:

Atividades ocupacionais:

6. Rotinas diárias:

6.1. Higiene oral: Feita pelo próprio idoso ( )

Feita pela equipe de enfermagem ( )

Outros ( )

Horário fixo ( )

a critério do idoso ( )

6.2. Sono: Horário fixo ( )

a critério do idoso ( )

\section{Alimentação:}

7.1. Restrições:

sal/açúcar ( ) consistência ( ) quantidade ( )

7.2. Dependência para alimentação:

alimentam-se sozinhos ( ) depende do idoso ( )

oferecida pela equipe de enfermagem ( ) oferecida por voluntários ( )

7.3. Local:

no refeitório ( ) no próprio quarto ( )

7.4. Postura durante a alimentação:

sentado ( ) deitado ( ) outros ( )

7.5. Postura após alimentação:

permanecem sentados ( ) deitam após alimentação ( )

7.6. Utilização de prótese dentária:

sim ( ) não ( )

7.7. Prazer em alimentar-se:

sim ( ) não ( )

Figura 1 - Questionário aplicado aos dirigentes de instituições asilares 


\begin{tabular}{|c|c|c|c|c|c|}
\hline Instituição & A & B & C & D & $\mathbf{E}$ \\
\hline \multicolumn{6}{|l|}{ Estrutura geral } \\
\hline Tipo de instituição & Privada & Filantrópica & Filantrópica & Filantrópica & Filantrópica \\
\hline Número de residentes & 20 & 45 & 36 & 53 & 20 \\
\hline $\begin{array}{l}\text { Critérios para } \\
\text { admissão na } \\
\text { instituição }\end{array}$ & Sem restrições & independentes & independentes & $\begin{array}{l}\text { Independentes } \\
\text { e acamados }\end{array}$ & Sem restrições \\
\hline Regime de saída & sozinhos & $\begin{array}{c}\text { Com } \\
\text { acompanhante }\end{array}$ & $\begin{array}{c}\text { Com } \\
\text { acompanhante }\end{array}$ & $\begin{array}{c}\text { Com } \\
\text { acompanhante }\end{array}$ & sozinhos \\
\hline Sexo dos residentes & Misto & Fem & Misto & Misto & Fem \\
\hline Faixa etária & Qualquer idade & $>60$ & $>60$ & $>60$ & $>60$ \\
\hline \multicolumn{6}{|l|}{ Recursos humanos } \\
\hline Médico & $\begin{array}{c}1 \text { vez por } \\
\text { semana } \\
\text { Clinico geral }\end{array}$ & $\begin{array}{c}1 \text { vez por } \\
\text { semana clinico } \\
\text { geral e geriatra }\end{array}$ & $\begin{array}{l}1 \text { vez por mês } \\
\text { médico }\end{array}$ & $\begin{array}{c}3 \text { vezes por } \\
\text { semana clinico }\end{array}$ & $\begin{array}{c}2 \text { vezes por } \\
\text { semana clinica } \\
\text { médica e } \\
\text { cardiologia }\end{array}$ \\
\hline Enfermagem & 24 horas & 24 horas & $\begin{array}{l}2 \text { vezes/ } \\
\text { semana }\end{array}$ & 24 horas & $\mathrm{N}$ \\
\hline Fisioterapeuta & $\mathrm{N}$ & S-n & $\mathrm{N}$ & S-n & S-n \\
\hline $\begin{array}{l}\text { Terapeuta } \\
\text { Ocupacional }\end{array}$ & $\mathrm{N}$ & $\mathrm{N}$ & $\mathrm{N}$ & $\mathrm{N}$ & S-n \\
\hline Serviço Social & $\mathrm{N}$ & $\mathrm{N}$ & $\mathrm{N}$ & $\begin{array}{l}6 \text { vezes por } \\
\text { semana }\end{array}$ & $\mathrm{N}$ \\
\hline Psicólogo & $\mathrm{N}$ & $\mathrm{N}$ & $\mathrm{N}$ & $\begin{array}{l}2 \text { vezes por } \\
\text { semana }\end{array}$ & $\mathrm{N}$ \\
\hline Dentista & $\mathrm{N}$ & $\mathrm{N}$ & $\mathrm{N}$ & $\begin{array}{l}1 \text { vez por } \\
\text { semana }\end{array}$ & $\mathrm{N}$ \\
\hline Nutricionista & $\begin{array}{l}3 \text { vezes por } \\
\text { semana }\end{array}$ & $\begin{array}{l}1 \text { vez por } \\
\text { semana }\end{array}$ & $\mathrm{N}$ & $\begin{array}{l}3 \text { vezes por } \\
\text { semana }\end{array}$ & $\mathrm{N}$ \\
\hline Fonoaudiólogo & $\mathrm{N}$ & $\mathrm{N}$ & $\mathrm{N}$ & $\mathrm{N}$ & $\mathrm{N}$ \\
\hline \multicolumn{6}{|l|}{ Rotina Alimentar } \\
\hline Higiene oral & $\begin{array}{l}\text { Sozinhos ou } \\
\text { auxiliar de } \\
\text { enfermagem }\end{array}$ & $\begin{array}{l}\text { Sozinhos ou } \\
\text { auxiliar de } \\
\text { enfermagem }\end{array}$ & $\begin{array}{l}\text { Sozinhos ou } \\
\text { auxiliar de } \\
\text { enfermagem }\end{array}$ & $\begin{array}{l}\text { Sozinhos ou } \\
\text { auxiliar de } \\
\text { enfermagem }\end{array}$ & $\begin{array}{l}\text { Sozinhos ou } \\
\text { auxiliados por } \\
\text { funcionários }\end{array}$ \\
\hline $\begin{array}{l}\text { Momento da higiene } \\
\text { oral }\end{array}$ & $\begin{array}{c}\text { Ao acordar } \\
\text { Após as } \\
\text { refeições }\end{array}$ & $\begin{array}{l}\text { Após as } \\
\text { refeições }\end{array}$ & $\begin{array}{l}\text { Após as } \\
\text { refeições }\end{array}$ & $\begin{array}{l}\text { Após as } \\
\text { refeições }\end{array}$ & $\begin{array}{l}\text { Após as } \\
\text { refeições }\end{array}$ \\
\hline $\begin{array}{l}\text { Consistências } \\
\text { Alimentares }\end{array}$ & $\begin{array}{l}\text { De acordo com } \\
\text { a aceitação }\end{array}$ & $\begin{array}{l}\text { Pastoso para } \\
\text { os debilitados }\end{array}$ & $\begin{array}{l}\text { Refeição igual } \\
\text { para todos }\end{array}$ & $\begin{array}{l}\text { Pastoso para } \\
\text { os debilitados }\end{array}$ & $\begin{array}{l}\text { Pastoso para } \\
\text { os debilitados }\end{array}$ \\
\hline $\begin{array}{l}\text { Independência } \\
\text { Alimentar }\end{array}$ & $\begin{array}{l}\text { Todos } \\
\text { auxiliados }\end{array}$ & $\begin{array}{c}\text { Todos } \\
\text { auxiliados }\end{array}$ & sozinhos & $\begin{array}{c}\text { Auxilio para os } \\
\text { acamados }\end{array}$ & $\begin{array}{l}\text { Todos } \\
\text { auxiliados }\end{array}$ \\
\hline $\begin{array}{l}\text { Posição após a } \\
\text { refeição }\end{array}$ & $\begin{array}{c}\text { Permitido deitar } \\
\text { após } 30 \\
\text { minutos }\end{array}$ & $\begin{array}{c}\text { Deitam ou } \\
\text { dormem após a } \\
\text { refeição }\end{array}$ & $\begin{array}{c}\text { Deitam ou } \\
\text { dormem após a } \\
\text { refeição }\end{array}$ & $\begin{array}{c}\text { Deitam ou } \\
\text { dormem após a } \\
\text { refeição }\end{array}$ & $\begin{array}{c}\text { Permitido deitar } \\
\text { após } 30 \\
\text { minutos }\end{array}$ \\
\hline Próteses dentárias & $\begin{array}{c}\text { Maioria com } \\
\text { próteses } \\
\text { dentárias }\end{array}$ & $\begin{array}{l}\text { Maioria com } \\
\text { próteses } \\
\text { dentárias }\end{array}$ & $\begin{array}{c}\text { Maioria sem } \\
\text { próteses } \\
\text { dentárias }\end{array}$ & $\begin{array}{c}\text { Maioria sem } \\
\text { próteses } \\
\text { dentárias } \\
\end{array}$ & $\begin{array}{l}\text { Maioria com } \\
\text { próteses } \\
\text { dentárias }\end{array}$ \\
\hline Prazer ao se alimentar & $\begin{array}{l}\text { Não se } \\
\text { queixam }\end{array}$ & $\begin{array}{l}\text { Não se } \\
\text { queixam }\end{array}$ & $\begin{array}{l}\text { Não se } \\
\text { queixam }\end{array}$ & $\begin{array}{l}\text { Não se } \\
\text { queixam }\end{array}$ & $\begin{array}{l}\text { Não se } \\
\text { queixam }\end{array}$ \\
\hline
\end{tabular}

Legenda: S: Sim N:Não S-n: se necessário

Figura 2 - Características das instituições 


\section{Estrutura (Recursos materiais)}

No que se refere ao caráter das instituições analisadas, apenas a instituição $A$ era privada. As demais $(B, C, D, E)$ eram filantrópicas e recebiam donativos da própria família dos idosos, de acordo com a condição financeira de cada um. Também eram mantidas com a ajuda de doações de entidades religiosas, contribuições de particulares e pequenas empresas. A instituição D (filantrópica) era a única que possuia convênio com a prefeitura do município do Rio de Janeiro.

A quantidade de idosos por instituição variou de acordo com o espaço físico de cada local. As instituições $A$ e $E$ eram residências que foram devidamente adaptadas e reestruturadas para abrigarem os idosos. Estas comportavam cerca de 20 idosos cada uma. As instituições $B, C$ e $D$ eram locais maiores e comportavam 45, 36 e 53 idosos respectivamente.

\section{Processos de admissão e cuidado}

Sexo e Idade: Quando questionados sobre o critério de elegibilidade para aceitação dos idosos, os asilos B e E só aceitavam mulheres. Os demais admitiam indivíduos de ambos os sexos. Levando em consideração a idade mínima permitida, com exceção da instituição $A$, que aceitava pessoas de todas as idades, todas as outras só recebiam indivíduos acima de 60 anos.

As instituições A e E não apresentavam restrições quanto ao nível de dependência dos idosos, aceitando inclusive pessoas com doenças degenerativas restritas ao leito. Os asilos $\mathrm{B}$ e $\mathrm{C}$ só admitiam idosos independentes, apesar de alguns deles serem auxiliados em determinadas funções na instituição B. No asilo D, a prefeitura era responsável pela triagem para eleger os idosos, que na maioria eram independentes, porém também abrigavam alguns acamados.

Das cinco instituições visitadas, três delas, $A, C$ e $D$, permitiam que os idosos independentes saíssem sozinhos. Já nas outras duas, mesmo aqueles que possuíam condições físicas para tal, só podiam sair acompanhados de algum familiar.

\section{Recursos Humanos}

Quanto aos recursos humanos disponíveis, todas as instituições apresentavam déficits de profissionais:

A) Médicos: todas as instituições tinham um médico responsável, porém, com freqüência semanal diferenciada em cada local. $\mathrm{Na}$ instituição $D$, o médico clínico geral visitava os idosos três vezes por semana. No asilo A, as visitas eram feitas uma vez por semana também por um clínico geral. $\mathrm{Na}$ instituição
$B$, a frequência era a mesma da instituição $A$, porém o médico responsável tinha formação em clínica geral e geriatria. No asilo E, o médico com especialização em clínica médica e cardiologia acompanhava os idosos quinzenalmente. $\mathrm{Na}$ instituição $\mathrm{C}$, a visita médica era realizada apenas uma vez por mês e o dirigente do local não soube informar a especialização do médico.

B) Enfermagem: as instituições A, B e D possuíam técnicos no período de 24 horas. Na instituição $C$, foi relatada a presença de auxiliares de enfermagem com freqüência de duas vezes semanais. Já o asilo E, não possuía nenhum profissional desta área.

C) Assistente Social: $A$ instituição $D$ foi a única que possui assistente social, com frequência de seis vezes por semana;

D) Psicólogo: duas vezes por semana na instituição D. As outras instituições não possuíam psicólogo.

E) Dentista: uma vez por semana na instituição $D$. As outras instituições não possuíam dentista.

F) Nutrição: Os asilos A e D apresentavam assistência nutricional três vezes semanais. $O$ asilo B apenas uma vez por semana e as outras instituições não contavam com esse trabalho.

G) Fisioterapia: Os idosos dos asilos B e D faziam tratamento fisioterápico de acordo com a necessidade específica de cada um.

H) Fonoaudiólogo: Nenhum dos asilos possuía fonoaudiólogo.

I) Terapeuta Ocupacional: Como o diretor da Instituição E era fisioterapeuta e terapeuta ocupacional, fazia as terapias se necessário. As outras instituições não possuíam esse profissional.

Segundo a direção da instituição $D$, os recursos humanos oferecidos e a frequência de cada profissional eram controlados pela prefeitura do município, elegendo quais profissionais eram necessários dentro desta instituição asilar. Nos outros locais esse critério foi estabelecido pelo próprio dirigente.

Vale salientar que, sendo o dirigente da instituição $E$ fisioterapeuta e terapeuta ocupacional aposentado, o mesmo realizava sessões terapêuticas nestas áreas com os idosos com alterações motoras. Nesta mesma instituição, os idosos participavam de atividades ocupacionais como musicoterapia e bailes dançantes, que ocorriam uma vez por mês.

\section{Rotinas de alimentação (cuidados orais)}

Referente à rotina de alimentação foi questionado o aspecto da higiene oral, em todas as instituições, os idosos considerados independentes a 
faziam sozinhos. Aqueles que não tinham condições para tal eram auxiliados por alguém da equipe de enfermagem. No caso da instituição $E$, que não possuía esse serviço, os idosos eram auxiliados por funcionários, que segundo o dirigente do local, eram treinados pelo mesmo para realização de tais tarefas.

O responsável da instituição $D$ relatou que, nos dias em que acontecia algum tipo de festividade na instituição ou quando muitos idosos encontravamse doentes, os técnicos de enfermagem tinham um número maior de tarefas para cumprir. Consequentemente, não era possível ajudar os idosos durante a higiene oral, ficando muitos deles sem realizar tal procedimento. Em todos os locais pesquisados, a higiene oral era realizada sempre após cada refeição. $\mathrm{Na}$ instituição $\mathrm{A}$, esse procedimento também era feito pela manhã ao acordar.

Ainda ao que se refere à rotina diária, na instituição E, os idosos possuíam um horário fixo para dormir, o que ocorria geralmente às 22 horas, logo após a ceia. Em todas as outras, isso ficava a critério de cada um, porém, a maioria dormia no mesmo horário.

No item referente à restrição alimentar segundo o critério consistência do alimento, todos os asilos procuravam elaborar um cardápio com pouca quantidade de sal e açúcar. Com relação à consistência dos alimentos, as instituições B, D e E ofereciam alimentação pastosa para todos os idosos mais debilitados. No asilo A houve diferença também na consistência alimentar de alguns idosos, porém, essa diferenciação foi feita de acordo com a aceitação de cada indivíduo. $A$ instituição $C$ foi a única a oferecer a mesma refeição quanto ao tipo de alimento e quantidade para todos os idosos indiscriminadamente, sem haver qualquer restrição alimentar.

Quando os dirigentes foram questionados sobre a independência no momento da alimentação, nos asilos $A, B$ e E, mesmo os idosos que comiam sozinhos, eram supervisionados e auxiliados. $\mathrm{Na}$ instituição $D$, a equipe de enfermagem auxiliava apenas os indivíduos acamados. Já no asilo $\mathrm{C}$, os idosos alimentavam-se sozinhos no refeitório, sem qualquer auxílio. Todos os dirigentes relataram que os idosos eram orientados a ficar sentados durante as refeições.

O responsável do asilo $A$ fez questão de afirmar que existe preocupação quanto à alimentação dos idosos, uma vez que já houve casos de pneumonia aspirativa. Segundo o mesmo, apenas após este fato, houve mudança quanto ao tipo de alimentos oferecido. Além disso, também relatou que desde então passou a ser mais rigoroso com a equipe de enfermagem, já que presenciou alguns episódios de engasgo e tosse por oferta inadequada da alimentação.

Logo após as refeições, os idosos dos asilos B, $C$ e $D$, costumavam deitar e dormir por algumas horas. Nas outras duas instituições, os responsáveis informaram que só permitiam que os idosos deitassem após meia hora do término da refeição.

Considerando a utilização de prótese dentária, os resultados variaram de acordo com o local. Nas instituições A, B e E, a maioria dos idosos faziam uso de prótese dentária, já nos outros dois lugares o contrário foi relatado, ou seja, os idosos não as possuía.

O último item do questionário referiu-se ao prazer com o ato de se alimentar e em todos os locais, os responsáveis disseram que os idosos não costumam apresentar queixa dos alimentos oferecidos.

\section{DISCUSSÃO}

A população idosa por todo o mundo vem crescendo exponencialmente. Um dos grandes futuros desafios para os profissionais da área da saúde é sem dúvida entender com clareza a fisiologia do organismo na terceira idade, transformações emocionais, possíveis adaptações a utensílios, meios de transporte, acessos a serviços, atendimento especializado entre outras necessidades do idoso. As entrevistas realizadas nessas instituições asilares mostraram a necessidade de cuidados especiais em relação à estrutura geral, recursos humanos e rotina alimentar dessas instituições, uma vez que foram identificados fatores de risco para a alimentação por via oral.

Em relação à estrutura geral dos asilos foi observado nas instituições pesquisadas, que a maioria tem caráter filantrópico e é carente de recursos materiais e humanos. Cada instituição cuida no mínimo de vinte no máximo de cinqüenta e três pessoas, sendo, portanto instituições relativamente pequenas, construídas em antigas residências reformadas.

Há uma preocupação evidente com a adaptação do espaço físico. Apenas uma instituição aceita asilados de qualquer idade, apesar de mesmo assim serem poucos abaixo de 60 anos, as outras quatro instituições só aceitam pacientes com 60 anos ou mais. Em duas instituições há uma restrição clara para o ingresso na instituição: o idoso tem que ser independente. Nas outras três instituições pacientes acamados, dependentes e com doenças diversas também são atendidos, o que justifica uma série de adaptações necessárias para as atividades de vida diária. 
Em relação aos recursos humanos oferecidos pelas instituições, nenhuma das instituições pesquisadas se enquadra na portaria $n^{\circ} 810 / 89$ que rege sobre as normas para funcionamento de casas de repouso, clínica e outras instituições destinadas ao atendimento de idosos, publicada no diário oficial de 27 de setembro de 1989. Esta inclui a necessidade de assistência médica, odontológica, nutricional, farmacêutica, psicossocial, de enfermagem e reabilitação, abrangendo fisioterapia, fonoaudiologia e terapia ocupacional. Essa carência já foi discutida por outros autores, visto que há dificuldade de encontrar asilos que mantenham uma equipe completa para assistência social e saúde do idoso ${ }^{14,26}$.

Nenhuma instituição apresentou o quadro de funcionários sugerido, uma delas inclusive não tinha nem auxiliares de enfermagem e a visita médica era realizada de quinzenalmente. Apenas uma instituição contemplava médico, enfermeiros, dentista, fisioterapeuta, assistente social, psicólogo e nutricionista em freqüências de 1 a 3 vezes por semana, mas não possuía fonoaudiólogos e terapeutas ocupacionais.

Além disso, é de grande importância que estes profissionais que fazem parte da equipe tenham conhecimento sobre as alterações fisiológicas que ocorrem durante o envelhecimento, participando de capacitação e educação continuada Tal fato possibilitaria conhecer e compreender não apenas o potencial, mas as limitações do idoso, principalmente durante a alimentação, tema foco dessa pesquisa. É importante ressaltar que não é necessário apenas uma equipe multiprofissional, mas esta tem que ser de fato interdisciplinar.

Em relação à rotina alimentar foram questionados aspectos específicos da alimentação e também da higienização oral, que se mostrou relacionada aos momentos de refeição. Em todas as instituições foi relatado que a higiene oral era realizada após cada refeição. Mas alguns dirigentes admitem que esta não se processe adequadamente por falta de recursos humanos.

A má higienização oral, que foi observada nos resultados, pode contribuir para o agravamento da inflamação de dentes e gengiva, podendo tornar o tecido bucal patogênico e acarretar em pneumonia caso o material de região orofaríngea seja broncoaspirado ${ }^{29,30}$.

Já, especificamente no que concerne a alimentação, ingestão oral de alimentos, são encontrados na literatura, trabalhos que evidenciam as diferenças naturais que vão ocorrer na deglutição durante $o$ envelhecimento. Uma das principais alterações fisiológicas ocorridas no indivíduo idoso é o aumento do tempo do estágio oral. Essa bradicinesia pode estar relacionada à diminuição de força ${ }^{27} \mathrm{e} a$ dificuldade de preparação oral do bolo alimentar, ocasionado entre outros fatores, pela perda natural do dentes, o que irá prejudicar diretamente a etapa mastigatória ${ }^{18,19} \mathrm{Em}$ três instituições a maioria dos idosos estava usando próteses dentárias, mas não se sabe as condições de adaptação e nas outras duas, a maioria não utilizava próteses, caracterizando mais um fator de risco para dificuldade de deglutição.

Em pesquisa realizada com idosos de duas instituições, sendo uma da rede pública e outra da rede privada de Recife, foi constatado que a maioria dos idosos deu preferência por alimentos macios em ambos os locais ${ }^{28}$. No entanto, no presente trabalho, verificou-se que na instituição em que a maior parte dos residentes não fez uso de prótese dentária, não houve qualquer tipo de restrição alimentar durante as refeições. Além disso, a adaptação da prótese dentária não costuma ocorrer de forma satisfatória em indivíduos idosos ${ }^{20}$. Considerando essa informação, isso leva a acreditar que mesmo aqueles que faziam uso de prótese dentária também poderiam apresentar alteração no processo de mastigação.

Outro problema encontrado na rotina asilar foi que muitos idosos dormiam logo após a refeição, com exceção de duas instituições. Deitar após a refeição pode lentificar o trânsito esofágico, o que favorece a ocorrência de refluxo gastroesofágico nessa população ${ }^{1}$. Caso haja episódios repetidos do RGE, pode favorecer o aparecimento de alterações anatômicas como a estenose esofágica ${ }^{13}$.

Para auxiliar esse processo o momento da refeição deve ser monitorado. Nesse estudo em uma das instituições não há auxiliares nesse momento e em outra o auxílio é oferecido apenas aos doentes acamados. Interessante considerar nesse momento que estudo ${ }^{31}$ já demonstrou $90 \%$ de incidência de disfagia em casas de repouso e evidenciou que as dietas poderiam ser oferecidas com progressão de consistências em alguns idosos e em outros deveria ser restrita, mostrando a clara necessidade de avaliação especializada da deglutição nessas instituições.

De uma maneira geral, nota-se que as modificações dos hábitos alimentares que surgem de forma natural e gradativa no idoso, como forma de adaptação às modificações das estruturas estomatognáticas, não são respeitadas pelas instituições asilares. Além disto, é inegável que essa população esteja mais suscetível a descompensação do mecanismo de deglutição, porém, conseguem adaptar-se a essas mudanças de forma que essas alterações não comprometem a saúde do individuo ${ }^{23}$. 
Assim torna-se possível pensar que a falta de estrutura e preparo desses abrigos pode ocasionar conseqüências mais graves na saúde geral do idoso asilado, quando comparado ao idoso não asilado ${ }^{3,24,25}$. Portanto, algumas condições próprias da instituição asilar, associadas ao estado emocional em que esses idosos se encontram quando estão internados nestes locais, podem tornar-se um fator com impacto negativo nas mudanças fisiológicas na senescência.

Ao término da pesquisa, pode-se notar que a instituição que apresenta condições mais próximas do ideal é a de caráter privado, principalmente no que diz respeito à alimentação. Pode-se observar que esta foi a única que apresentou um dirigente consciente sobre a importância de manter uma alimentação segura. Mesmo assim, não tinham equipe interdisciplinar necessária ao adequada ao atendimento completo.

Pelo que se pode observar a maioria das instituições de caráter filantrópico ainda são bastante deficitárias quando se trata da saúde global do indivíduo idoso. Isto ressalta não só a importância e a necessidade da atuação do fonoaudiólogo, do dentista, do psicólogo, junto a estas instituições, mas também a necessidade de observância das resoluções de políticas públicas que já existem e que estabeleceram condições de estrutura mínima material e humana para o funcionamento dessas instituições.

Os resultados obtidos no presente estudo têm grande importância na medida em que remetem a realização de novos estudos por meio de avaliações clínicas e ou instrumentais direcionadas aos próprios idosos.

\section{CONCLUSÃO}

Pode-se concluir que, de acordo com a amostra pesquisada, as instituições asilares não estão preparadas para promover ao idoso as condições ideais que garantam uma saúde global. A escassez dos recursos humanos exigidos e a falta de capacitação dos profissionais envolvidos na rotina de alimentação tendem a potencializar as chances de ocorrência de alterações no processo de deglutição. Contudo, com exceção de um, todos os representantes dos asilos negaram qualquer tipo de intercorrência durante as refeições.

\begin{abstract}
Purpose: to check if the conditions related to general structure, human resources and daily routine of feeding in asylums can increase the chances for an alteration in the deglutition process of the elderlies. Methods: a questionnaire was elaborated to be applied to the directors of five institutions in the city of Rio de Janeiro. Results: it can be observed that the sole factor related to the structure of the place that had relevance in the obtained results was the nature of the institution, as the private system was the only one that was considered near the ideal conditions. Regarding the existing human resources, none of the institutions had all the professionals demanded by the Ordinance no810/89. In relation to the daily feeding routines, in one of the investigated institutions, where the majority of the residents did not use denture, there was no restriction as for the offered food consistency. Another significant factor was the carelessness in terms of oral hygiene, favoring the settling of bacteria in the mouth, which can cause serious consequences in case of microaspirations. In three of the investigated institutions, it was observed that the elderlies lay down to sleep soon after the meals, fact that also had great importance, as this favors the incidence of gastroesophageal reflux episodes that can lead to gradual dysphagia. Conclusion: despite the fact that the asylum directors did not mention any referring problem in the process of deglutition of the elderlies, they show proper characteristics that can increase the possibilities for occurrence of alterations in the deglutition of these elderlies.
\end{abstract}

KEYWORDS: Asylum; Aged; Deglutition Disorders 


\section{REFERÊNCIAS}

1. Silva FML, Neves SRPC, Silva MMML. Processo de envelhecimento e suas alterações nos diversos sistemas orgânicos. JBM. 2005; 88(4):32-6.

2. Christensen K, Doblhammer G, Rau R, Vaupel JW. Ageing populations: the challenges ahead. Lancet, 2009; 374: 1196-208..

3. Roy N,Stemple J, MerrillRM, Thomas L. Dysphagia in the Elderly: Preliminary Evidence of Prevalence, Risk Factors, and Socioemotional Effects. Annals of Otology, Rhinoiogy \& Laryngology 2007;116(11):858-865.

4. Freitas MIA, Ribeiro AF, Chiba T, Mansur LL. Investigação fonoaudiológica de idosos em programa de assistência domiciliar - Revista Einstein. 2007; 5(1):6-9

5. Bottino-BravoP, and Thomson J. When It's a Hard Act to Swallow: Dysphagia in Home Care. Home Healthcare Nurse, 2008; 26(4): $244-50$.

6. McCullough GH, Rosenbek JC, Wertz RT:Defining swallowing function by age. TopGeriatr Rehabil, 2007; 23: 290-307.

7. Leder S. e Suiter DM. An Epidemiologic Study on Aging and Dysphagia in the Acute Care Hospitalized Population: 2000-2007.. Gerontology 2009;55:714-8.

8. Cabre M, Serra-Prat M, Palomera E, Almirall J, Pallares R, Clavé P. Prevalence and prognostic implications of dysphagia in elderly patients with pneumonia. Age and Ageing , 2010; 39: 39-45.

9. Allepaerts S; Delcourt S; Petermans J. Swallowing disorders in the elderly: an underestimated problem. Rev Med Liege; 2008. 63(12):715-21.

10. Ney D, Weiss J, Kind A, Robbins J. Senescent Swallowing: Impact, Strategies and Interventions. Nutr Clin Pract. 2009 ; 24(3): 395-413.

11. Pauly L, Stehle $P$, Volkert $D$. Nutricional situation of elderly nursing home residents. Z Gerontology Geriatric. 2007; 40:3-12.

12. Tanton M. Developing a screening tool and training package to identify dysphagia in all settings. Nurs Times, 2010; 106(15):18-20.

13. Germain I,Dufresne T, Gray-Donald K. A Novel Dysphagia Diet Improves the Nutrient Intake of Institutionalized Elders. J Am Diet Assoc. 2006;106:1614-23.

14. Dias BKP, Cardoso MCAF. Características da função de deglutição em um grupo de idosas institucionalizadas. Estud. Interdiscipl. Envelhec. 2009; Porto Alegre, 14(1): 107-24.

15. Wakasugi $Y$, Tohara $H$, Hattori $F$, Motohashi $Y$, Nakane A, Goto S, Ouchi Y, Mikushi S, Takeuchi S, Uematsu H. Screening Test for Silent Aspiration at the Bedside. Dysphagia 2008; 23(4):364-70.
16. Gaião LR, Almeida MEL, Heukelbach J. Perfil epidemiológico da cárie dentária, doença periodontal, uso e necessidade de prótese em idosos residentes em uma instituição na cidade de Fortaleza, Ceará. Rev Bras Epidemiol. 2005; 8(3):316-23.

17. Montal S, Tramini P, Triay JA and Valcarcel $J$. Oral hygiene and the need for treatment of the dependent institutionalised elderly. Gerodontology. 2006; 23; 67-72.

18. Amaral AKFJ, Silva HJ, Cabral ED. Fatores determinantes do tempo de maceração dos alimentos em idosas edêntulas totais. Rev CEFAC, 2009; 11(3), 398-404.

19. Humbert ID, Robbins J. Dysphagia in the Elderly. Phys Med Rehabil Clin N Am.2008;19(3): 853-66.

20. Yamada EK, Siqueira KO, Xerez D, Koch HA, Costa MM. The influence of oral and pharyngeal phases on the swallowing dynamic. Arq Gastroenterol. 2004; 41(1):18-23.

21. Leslie P, Drinnan MJ, Ford GA, Wilson JA. Swallow respiratory patterns and aging: presbyphagia or dysphagia. J Gerontol series A: Biol Sci Med Sci. 2005; 60:391-5.

22. Fukunaga $A$, Uematsu $H$, Sugimoto $K$. Influences of aging on taste perception and oral somatic sensation. J Gerontol A Biol Sci Med Sci 2005;60(1):109-13.

23. White GN,. O'Rourke F, Ong BS, Cordato DJ, Chan DKY. Dysphagia: causes, assessment, treatment and management. Geriatrics, 2008; 63(5): 15-20.

24. Lima RMF, Amaral AKFJ, Aroucha EBL, Vasconcelos MJ, Silva HJ, Cunha DA. Adaptações na mastigação, deglutição e fonoarticulação em idosos de instituição de longa permanência. Rev CEFAC, 2009;11(supl3):405-22.

25. Eisenstadt ES. Dysphagia and aspiration pneumonia in older adults. Journal of the American Academy of Nurse PractitionerS, 2010; 22(1): 17-22.

26. Ferreira RC; Magalhães CS; Rocha ES; Schwambach CW; Moreira AN. Saúde bucal de idosos residentes em instituiçoes de longa permanência de Belo Horizonte, Minas Gerais, Brasil. Cad Saude Publica; 2009; 25(11):2375-85.

27. Fazito LT, Perim JV, Di Ninno CQMS. Comparação das queixas alimentares de idosos com e sem prótese dentária. Rev. CEFAC. 2004; 6(2):143-50.

28. Queiroz CM; Rezende CM; Molena CCL; Denardin OVP; Rapoport A. Avaliação da condição periodontal no idoso. Rev. Bras. Cir. Cabeça Pescoço; 2008; 37(3):156-9.

29. Kullberg EE, Sjögren P, Forsell M, Hoogstraate J, Herbst B \& Johansson O. Dental hygiene education 
for nursing staff in a nursing home for older people. Journal of Advanced Nursing. 2010; 66(6),1273 - 9. 30. Drinka P. Preventing Aspiration in the Nursing Home: The Role of Biofilm and Data from the ICU. J Am Med Dir Assoc 2010; 11(1): 70-7.
31. Ghroer, M, McKaig. T.N. Dysphagia and dietary levels in skilled nursing facilities. J. Am. Geriatr. Soc., 1995;43:538.

RECEBIDO EM: 23/05/2010

ACEITO EM: 04/10/2010

Endereço para correspondência:

Ana Maria Furkim

Rua São Mateus, 175

São Paulo - SP

CEP: 04721-020

Email: ana.furkim@gmail.com 